\title{
Logic and Model Checking for Hidden Markov Models`
}

\author{
Lijun Zhang ${ }^{1}$, Holger Hermanns ${ }^{1,2}$, and David N. Jansen ${ }^{2}$ \\ ${ }^{1}$ Department of Computer Science, Saarland University, \\ D-66123 Saarbrücken, Germany \\ ${ }^{2}$ Department of Computer Science, University of Twente, \\ Enschede, The Netherlands
}

\begin{abstract}
The branching-time temporal logic PCTL* has been introduced to specify quantitative properties over probability systems, such as discrete-time Markov chains. Until now, however, no logics have been defined to specify properties over hidden Markov models (HMMs). In HMMs the states are hidden, and the hidden processes produce a sequence of observations. In this paper we extend the logic PCTL $^{*}$ to POCTL*. With our logic one can state properties such as "there is at least a 90 percent probability that the model produces a given sequence of observations" over HMMs. Subsequently, we give model checking algorithms for POCTL* over HMMs.
\end{abstract}

\section{Introduction}

Hidden Markov models (HMMs) [17] were developed in the late 1960's and have been proven to be very important for many applications, especially speech recognition [13], character recognition [22], biological sequence analysis [5], and protein classification problems [15]. Lately, HMMs receive increased attention in the context of communication channel modelling [20] and of QoS properties in wireless networks [9].

An HMM is a doubly embedded stochastic process with an underlying stochastic process over some state space, which is hidden. The occupied state can only be observed through another set of stochastic processes that produce a sequence of observations. Given the sequence of observations, we do not exactly know the occupied state, but we do know the probability distribution over the set of states. This information is captured by a so-called belief state.

For a given HMM, one is often interested in the properties of the underlying stochastic process. In addition, one is also interested to reason about properties over the other set of stochastic processes which produce the observations. In this

\footnotetext{
* Parts of this work was carried out while the third author was with the Max-PlanckInstitut für Informatik, Saarbrücken. This work is partially supported by the NWODFG bilateral project VOSS, the NWO Vernieuwingsimpuls award 016.023.010, and by the DFG as part of the Transregional Collaborative Research Center SFB/TR 14 AVACS.
} 
paper, we introduce a logic called POCTL*, which consists of state formulas, path formulas and belief state formulas. POCTL* allows us to specify properties of interests over HMMs. We consider the property:

There is at least a 90 percent probability that the model produces the sequence of observations $O=\left(o_{0}, o_{1}, \ldots, o_{n}\right)$.

This property can be expressed in POCTL* by $\mathcal{P}_{\geq 0.9}\left(\mathbf{X}_{o_{0}} \mathbf{X}_{o_{1}} \ldots \mathbf{X}_{o_{n}} t t\right)$. As indicated by Rabiner [17], this probability can be viewed as the score which specifies how well a given model matches the observations. In Speech Recognition [13], we want to find out the most likely sentence (with the highest score) given a language and some acoustic input (observations). Assuming that we know that the HMM for the word "Need" produces the acoustic observations $O$ with probability at least 0.9 , then we can almost conclude that this acoustic input represents the word "Need". In the protein classification problem, we want to classify the new protein to one known class. The idea is to construct an HMM for every known class, and calculate the score of the new protein under every class. The new protein belongs to the class which matches it (produces it with the highest probability).

On one hand, POCTL* is basically an extension of PCTL* where the next operator is equipped with an observation constraint. On the other hand, POCTL* can also be considered as a variant of the temporal logic ACTL*, presented by De Nicola et al. [14], in which the usual next operator is extended to constrain the action label of the transition.

The PCTL* model checking $[2,1,11]$ problem can be reduced to the QLS (quantitative LTL specification) model checking problem. For QLS model checking, one constructs first a Büchi automaton for an LTL formula using well-known methods $[23,21,10]$, and then builds the product of the system and the constructed Büchi automaton. Finally, the QLS model checking problem can be reduced to a probabilistic reachability analysis in the product system.

Following the same line, we shall present the POCTL* model checking algorithm as follows. First, it will be reduced to the QOS (quantitative OLTL specification, where OLTL abbreviates Observational LTL) model checking problem. The latter can be further reduced to a probabilistic reachability analysis in the product automaton. To that end, we construct a Büchi automaton for a given OLTL formula. The construction is an adaption of the one presented in [10].

\section{Preliminaries}

Rabin Automaton. A deterministic Rabin automaton $[18,2]$ is a tuple $\mathcal{R}_{\phi}=$ $\left(\Sigma, Q, q_{i n}, \delta, U\right)$ where $\Sigma$ is a nonempty finite alphabet, $Q$ is a finite set of states, $q_{\text {in }} \in Q$ is the initial state, $\delta: Q \times \Sigma \rightarrow Q$ is the transition function, and $U=\left\{\left(P_{i}, R_{i}\right) \mid i=1, \ldots, r\right\}$ is the Rabin acceptance condition where $P_{i}, R_{i} \subseteq Q$.

We call an infinite sequence $w=w_{1}, w_{2}, \ldots$ over $\Sigma$ a word over $\Sigma$. $w$ induces an unique path $\pi=q_{0}, q_{1}, \ldots$ in $\mathcal{R}$ where $q_{0}=q_{i n}$, and $q_{i+1}=\delta\left(q_{i}, w_{i}\right)$ for $i=$ $0,1, \ldots \pi$ is an accepting path if $\inf (\pi) \subseteq P_{j}$ and $\inf (\pi) \cap R_{j} \neq \emptyset$ for some $j \in$ $\{1, \ldots, r\}$ where $\inf (\pi)$ denotes the set of states that occur infinitely often in $\pi$. 
Discrete-time Markov Chains. A labeled discrete-time Markov chain (DTMC) is a tuple $\mathcal{D}=(S, \mathbf{P}, L)$ where $S$ is a finite set of states, $\mathbf{P}: S \times S \rightarrow[0,1]$ is a probability matrix satisfying $\sum_{s^{\prime} \in S} \mathbf{P}\left(s, s^{\prime}\right) \in\{0,1\}$ for all $s \in S$, and $L: S \rightarrow 2^{A P}$ is a labeling function.

\section{Hidden Markov Models}

This section first recalls the concept of HMM, then defines belief states, paths over HMM, and probability spaces for a given HMM.

\subsection{Labeled Discrete-Time HMMs}

An HMM [17] is a doubly embedded stochastic process with an underlying stochastic process that is hidden, but can only be observed through another set of stochastic processes that produce a sequence of observations. We add a labeling function to the standard definition of HMMs, in other words, we consider an HMM as an extension of a labeled DTMC:

Definition 1. A labeled discrete-time $H M M \mathcal{H}$ is a tuple $(S, \mathbf{P}, L, \Theta, \mu, \alpha)$ where $(S, \mathbf{P}, L)$ is a labeled DTMC, $\Theta$ is a finite set of observations, $\mu: S \times \Theta \longrightarrow[0,1]$ is an observation function satisfying $\sum_{o \in \Theta} \mu(s, o)=1 \forall s \in S$, and $\alpha$ is an initial distribution on $S$ such that $\sum_{s \in S} \alpha(s)=1$.

The observation set $\Theta$ corresponds to the output of the model. By definition, $\mu(s, \cdot)$ is a distribution on $\Theta$, and $\mu(s, o)$ indicates the probability that the state $s$ produces the observation $o$. For the sake of brevity, we write $\mu_{s}(o)$ instead of $\mu(s, o)$. The probability that the model starts with state $s$ is $\alpha(s)$. In what follows we use the term HMM to refer to a labeled discrete-time HMM. For technical reasons, we assume there is no absorbing state in an HMM throughout our discussion ${ }^{1}$.

\subsection{Belief State}

The observation depends stochastically and exclusively on the current state. In general, the same observation could be emitted by several different states; therefore, we are uncertain about the current state, but, we can summarize the historical observations in a belief state (or information state) $[12,16]$ which is a distribution over $S$. A belief state is not really a state of the HMM. Rather, it is a way to describe what we know about the state, given the history of observations. The set of all possible belief states is called the belief space, and is denoted by $\mathcal{B}$. We use $S^{t}$ with $S^{t} \in S$ to denote the state at time $t$, and $O^{t} \in \Theta$ to denote the observation at time $t$. We write $b_{t}$ to denote the belief state at time $t$.

\footnotetext{
${ }^{1}$ As indicated by Baier [2] (for concurrent probabilistic systems), this is a harmless restriction since any system can be transformed into an "equivalent" system without absorbing states. For an HMM $\mathcal{H}$ with absorbing states, we insert just a special state $\dagger$ with a self-loop and transitions from any absorbing state in $\mathcal{H}$ to $\dagger$.
} 
Definition 2. Let $o_{i} \in \Theta$ where $i=0, \ldots, t$. The belief state $b_{t}$ at time $t$, is the distribution over $S$ at time $t$ given the observation history $o_{0}, \ldots, o_{t}$ :

$$
b_{t}(s)=P\left(S^{t}=s \mid O^{0}=o_{0}, \ldots, O^{t}=o_{t}, \mathcal{H}\right) \forall s \in S
$$

Now given the historical observations $o_{0}, \ldots, o_{t}$, the question is how to calculate the belief state $b_{n}$. The belief state at time 0 only depends on the initial distribution and the first observation. The belief state at time $t$ captures all of our information about the past. As a result, we can inductively calculate the current belief state $b_{t}$ based on the previous belief state $b_{t-1}$ and the current observation $o_{t}$. This is illustrated in Figure 1.

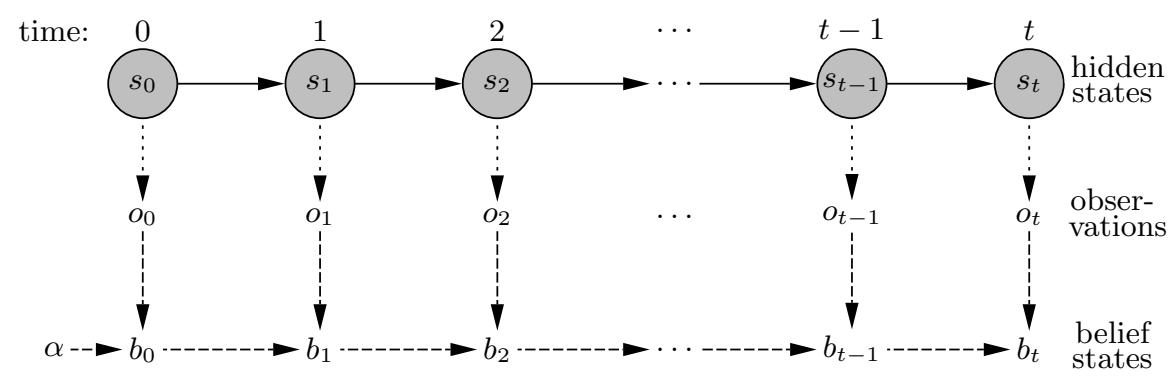

Fig. 1. Updating belief states

We depict the states in gray circles to indicate that they are hidden. The states together with the solid arrows between them represent the underlying state evolvement. The dotted arrows between states and observations mean that the observation $o_{t}$ is produced from the state $s_{t}$ according to the observation function $\mu$. As a particular case, $b_{0}$ is a function of $o_{0}$ and the initial distribution $\alpha$. Applying the Bayesian rule and the definition of $b_{0}$ we get: $b_{0}(s)=\frac{\alpha(s) \mu_{s}\left(o_{0}\right)}{K_{0}}$ where $K_{0}$ is a normalizing constant with value $\sum_{s \in S} \alpha(s) \mu_{s}\left(o_{0}\right)$.

The dashed arrows, between the current observation $o_{t}$, previous belief state $b_{t-1}$ and the current belief state $b_{t}$, mean that $b_{t}$ depends on $o_{t}$ and $b_{t-1}$ for all $t=1, \ldots, n$. Again, applying the Bayesian rule and the definition of $b_{t}$ we have: $b_{t+1}(s)=\frac{\sum_{s_{t} \in S} b_{t}\left(s_{t}\right) \mathbf{P}\left(s_{t}, s\right) \mu_{s}\left(o_{t+1}\right)}{K_{t+1}}$ where $K_{t+1}$ is a normalizing constant with value: $\sum_{s \in S}\left(\sum_{s_{t} \in S} b_{t}\left(s_{t}\right) \mathbf{P}\left(s_{t}, s\right) \mu_{s}\left(o_{t+1}\right)\right)$. Hence, given the historical observations, we are able to calculate the current belief state.

\subsection{Paths in HMM and Probability Spaces over Paths}

Given $\mathcal{H}=(S, \mathbf{P}, L, \Theta, \mu, \alpha)$, let $s_{i} \in S$ and $o_{i} \in \Theta$ for all $i \in \mathbb{N}$. A path $\sigma$ of $\mathcal{H}$ is a sequence $\left(s_{0}, o_{0}\right),\left(s_{1}, o_{1}\right) \ldots \in(S \times \Theta)^{\omega}$ where $\mu_{s_{i}}\left(o_{i}\right)>0, \mathbf{P}\left(s_{i}, s_{i+1}\right)>0$ for all $i \in \mathbb{N}$ and $(S \times \Theta)^{\omega}$ denotes the set of infinite sequences of elements of $S \times \Theta$. 
For a path $\sigma$ and $i \in \mathbb{N}$, let $\sigma_{s}[i]=s_{i}$ denote the $(i+1)$ st state of $\sigma$, and $\sigma_{o}[i]=o_{i}$ denote the $(i+1)$ st observation of $\sigma$. Let $\sigma[i]$ denote the suffix path of $\sigma$ starting with $\sigma_{s}[i]$, i. e., $\left(s_{i}, o_{i}\right),\left(s_{i+1}, o_{i+1}\right), \ldots$ Note that $\sigma[0]=\sigma$.

Let $\operatorname{Path}^{\mathcal{H}}$ denote the set of all paths in $\mathcal{H}$, and $\operatorname{Path}^{\mathcal{H}}(s)$ denote the set of paths in $\mathcal{H}$ that start in $s$. The superscript $\mathcal{H}$ is ommitted whenever convenient. We define a probability space on paths of $\mathcal{H}$ using the standard cylinder construction. For a path $\left(s_{0}, o_{0}\right),\left(s_{1}, o_{1}\right), \ldots$, we define the basic cylinder set induced by the prefix of this path as follows:

$$
\mathcal{C}\left(\left(s_{0}, o_{0}\right),\left(s_{1}, o_{1}\right), \ldots,\left(s_{n}, o_{n}\right)\right):=\left\{\sigma \in P a t h \mid \forall i \leq n . \sigma_{s}[i]=s_{i} \wedge \sigma_{o}[i]=o_{i}\right\}
$$

If it is clear from the context, we use just $\mathcal{C}$ to denote this cylinder set. $\mathcal{C}$ consists of all paths $\sigma$ starting with $\left(s_{0}, o_{0}\right),\left(s_{1}, o_{1}\right), \ldots\left(s_{n}, o_{n}\right)$. Let $\mathcal{C} y l$ contain all sets $\mathcal{C}\left(\left(s_{0}, o_{0}\right), \ldots,\left(s_{n}, o_{n}\right)\right)$ where $s_{0}, \ldots, s_{n}$ range over all state sequences and $o_{0}, \ldots, o_{n}$ range over all observation sequences. Let $\mathcal{F}$ be the $\sigma$-algebra on Path generated by $\mathcal{C} y l$. Let $\mathbf{i}\left(s, s_{0}\right)=1$ if $s=s_{0}$, and $\mathbf{i}\left(s, s_{0}\right)=0$ if $s \neq s_{0}$. The probability measure ${ }^{2} \operatorname{Pr}_{s}$ on $\mathcal{F}$ is defined by induction on $n$ by $\operatorname{Pr}_{s}\left(\mathcal{C}\left(s_{0}, o_{0}\right)\right)=$ $\mathbf{i}\left(s, s_{0}\right) \mu_{s_{0}}\left(o_{0}\right)$ and, for $n>0$ :

$$
\begin{aligned}
& \operatorname{Pr}_{s}\left(\mathcal{C}\left(\left(s_{0}, o_{0}\right), \ldots,\left(s_{n}, o_{n}\right)\right)\right) \\
& \quad=\operatorname{Pr}_{s}\left(\mathcal{C}\left(\left(s_{0}, o_{0}\right), \ldots,\left(s_{n-1}, o_{n-1}\right)\right)\right) \cdot \mathbf{P}\left(s_{n-1}, s_{n}\right) \mu_{s_{n}}\left(o_{n}\right)
\end{aligned}
$$

By induction on $n$, we obtain:

$$
\operatorname{Pr}_{s}\left(\mathcal{C}\left(\left(s_{0}, o_{0}\right), \ldots,\left(s_{n}, o_{n}\right)\right)\right)=\mathbf{i}\left(s, s_{0}\right) \mu_{s_{0}}\left(o_{0}\right) \prod_{i=1}^{n} \mathbf{P}\left(s_{i-1}, s_{i}\right) \mu_{s_{i}}\left(o_{i}\right)
$$

Lemma 3. Let $s \in S$. The triple $\left(\right.$ Path, $\left.\mathcal{F}, \operatorname{Pr}_{s}\right)$ on domain Path is a probability space, where $\mathcal{F}$ is the $\sigma$-algebra generated by the set of basic cylinder sets $\mathcal{C} y l$, and $\operatorname{Pr}_{s}$ is the probability measure which is described by Equation 1.

Let $b \in \mathcal{B}$ be a belief state, and $C \in \mathcal{C} y l$ be a basic cylinder set. We extend the probability measure with respect to a belief state $b$ by: $\operatorname{Pr}_{b}(C)=\sum_{s \in S} b(s)$. $\operatorname{Pr}_{s}(C)$. Similar to Lemma 3, the triple $\left(\right.$ Path, $\left.\mathcal{F}, \operatorname{Pr}_{b}\right)$ on domain Path is also a probability space.

\section{The Logic POCTL*}

This section presents the branching-time temporal logic Probabilistic Observation $\mathrm{CTL}^{*}$ (POCTL*) which allows us to specify properties over HMMs. We have indicated in the introduction that for an HMM, one wants to specify properties over the underlying DTMC and in addition, one is also interested in reasoning

${ }^{2}$ We define here actually a probability function $\operatorname{Pr}_{s}$ on the set $\mathcal{C} y l$. For $\mathcal{F}$ is a $\sigma$-algebra generated by $\mathcal{C} y l$, this probability function can be extended to a unique probability measure on $\mathcal{F}$. 
about properties over the other set of stochastic processes which produce observations. The logic PCTL* is interpreted over DTMCs to express quantitative stochastic properties $[2,7,6]$. We extend PCTL* to POCTL* such that the next operator is equipped with an observation constraint. In this way we can state properties over the observations, e.g., $X_{o} \phi$ means that the next observation is $o$ and the subsequent path satisfies $\phi$.

POCTL $^{*}$ can be also considered as a variant of the temporal logic ACTL* introduced by De Nicola et al. [14]. ACTL* is interpreted over Labeled Transition Systems (LTS) and has been proven to have the same power as CTL*. In ACTL* $^{*}$ the usual next operator is extended to interpret the labeled action of the transition (e.g., $X_{a} \phi$ means the next transition is labeled with an action $a$ and the subsequent path satisfies $\phi)$.

\subsection{Syntax of POCTL*}

Let $\mathcal{H}=(S, \mathbf{P}, L, \Theta, \mu, \alpha)$ be an HMM with $o \in \Theta$. The syntax of the logic POCTL* $^{*}$ is defined as follows:

$$
\begin{aligned}
\Phi & :=a|\neg \Phi| \Phi \wedge \Phi \mid \epsilon \\
\phi & :=\Phi|\neg \phi| \phi \wedge \phi\left|\mathbf{X}_{o} \phi\right| \phi \mathcal{U}^{\leq n} \phi \\
\epsilon & :=\mathcal{P}_{\unlhd p}(\phi)|\neg \epsilon| \epsilon \wedge \epsilon
\end{aligned}
$$

where $n \in \mathbb{N}$ or $n=\infty, 0 \leq p \leq 1$ and $\unlhd \in\{\leq,<, \geq,>\}$.

The syntax of POCTL* consists of state formula, path formula and belief state formula. As in $\mathrm{CTL}^{*}$, we use $\Phi, \Psi$ for state formula and $\phi, \psi$ for path formula. The formula $\epsilon$ is called belief state formula. In HMMs, we are uncertain about the current state, but we always know the current belief state. Therefore, we want to know if some (probabilistic) properties are valid in belief states. We consider the example in the introduction:

There is at least a 90 percent probability that the model produces a sequence of observations $O=\left(o_{0}, o_{1}, \ldots, o_{n}\right)$.

This can be expressed by a belief state formula $\epsilon=\mathcal{P}_{\geq 0.9}\left(\mathbf{X}_{o_{0}} \mathbf{X}_{o_{1}} \ldots \mathbf{X}_{o_{n}} t t\right)$. Intuitively, a belief state $b$ satisfies $\epsilon$ if the probability measure w.r.t. $b$, i. e., $\operatorname{Pr}_{b}$, of the set of paths satisfying $\mathbf{X}_{o_{0}} \mathbf{X}_{o_{1}} \ldots \mathbf{X}_{o_{n}} t t$ meets the bound $\geq 0.9$. In Speech Recognition [13], we want to find out the most likely sentence given a language and some acoustic input. For example, if we know that the HMM for the word "Need" produces the acoustic observations with probability at least 0.9 , we can almost conclude that this acoustic input represents the word "Need". We indicate that this property cannot be expressed by any sublogics of POCTL* that we shall define later.

For the sake of simplicity, we do not consider the exist operator. The formula $\exists \phi$ is almost equivalent to the probability formula $\mathcal{P}_{>0} \phi$. The standard (i.e., unbounded) until formula is obtained by taking $n$ equal to $\infty$, i. e., $\phi \mathcal{U} \psi=$ $\phi \mathcal{U} \leq \infty \psi$. We use the abbreviations $\wedge, \diamond, \square$ which are defined in the same way as for CTL*. The timed variants of the temporal operators can be derived, e.g., $\diamond{ }^{\leq n} \phi=t t \mathcal{U}^{\leq n} \phi, \square^{\leq n} \phi=\neg \diamond \leq n \neg \phi$. 


\subsection{Semantics of POCTL*}

Let $\mathcal{H}=(S, \mathbf{P}, L, \Theta, \mu, \alpha)$ be an HMM with $s \in S$ and $\sigma \in$ Path. The semantics of POCTL* is defined by a satisfaction relation (denoted by $\models$ ) either between a state $s$ and a state formula $\Phi$, or between a path $\sigma$ and a path formula $\phi$, or between a belief state $b$ and a belief state formula $\epsilon$. We write $\mathcal{H}, s \models \Phi$, $\mathcal{H}, \sigma \models \phi$ and $\mathcal{H}, b \models \epsilon$ if state $s$, path $\sigma$ and belief state $b$ satisfy state formula $\Phi$, path formula $\phi$ and belief state formula $\epsilon$, respectively. If the model $\mathcal{H}$ is clear from the context, we simply write $s \models \Phi, \sigma \models \phi$ and $b \models \epsilon$.

Let $b_{s}$ be the belief state with $b_{s}(s)=1$ and $b_{s}\left(s^{\prime}\right)=0$ for $s^{\prime} \neq s$. The satisfaction relation $\models$ is defined in Figure 2 where $\operatorname{Pr}_{b}\{\sigma \in$ Path $\mid \sigma \models \phi\}$, or $\operatorname{Pr}_{b}(\phi)$ for short, denotes the probability measure of the set of all paths which satisfy $\phi$ and start states weighted by $b$.

$\begin{array}{lll}s \models a & \text { iff } & a \in L(s) \\ s \models \neg \Phi & \text { iff } & s \not \models \Phi \\ s \models \Phi \wedge \Psi & \text { iff } & s \models \Phi \wedge s \models \Psi \\ s \models \epsilon & \text { iff } & b_{s} \models \epsilon \\ \sigma \models \Phi & \text { iff } & \sigma_{s}[0] \models \Phi \\ \sigma \models \neg \phi & \text { iff } & \sigma \not \models \phi \\ \sigma \models \phi \wedge \psi & \text { iff } & \sigma \models \phi \wedge \sigma \models \psi \\ \sigma \models \mathbf{X}_{o} \phi & \text { iff } & \sigma_{o}[0]=o \wedge \sigma[1] \models \phi \\ \sigma \models \phi \mathcal{U}^{\leq n} \psi & \text { iff } & \exists 0 \leq j \leq n .(\sigma[j] \models \psi \wedge \forall i<j . \sigma[i] \models \phi) \\ b \models \mathcal{P}_{\unlhd p}(\phi) & \text { iff } & \operatorname{Pr}_{b}\{\sigma \in \operatorname{Path} \mid \sigma \models \phi\} \unlhd p \\ b \models \neg \epsilon & \text { iff } & b \not \models \epsilon \\ b \models \epsilon \wedge \epsilon^{\prime} & \text { iff } & b \models \epsilon \wedge b \models \epsilon^{\prime}\end{array}$

Fig. 2. Semantics of POCTL*

A path satisfies the new operator $\mathbf{X}_{o} \phi$ if it starts with the observation $o$ and the suffix ${ }^{3} \sigma[1]$ satisfies $\phi$. Let $\Omega$ be a set of observations, i. e., $\Omega \subseteq \Theta$. We use the abbreviation $\mathbf{X}_{\Omega} \phi$ for $\bigvee_{o \in \Omega} \mathbf{X}_{o} \phi$ to shorten our notations.

By the definition of $\mathbf{X}_{\Omega} \phi$, we obviously have $\sigma \models \mathbf{X}_{\Omega} \phi$ iff $\sigma_{o}[0] \in \Omega \wedge \sigma[1] \models$ $\phi$. The usual next operator can be described as $\mathbf{X} \phi \equiv \mathbf{X}_{\Theta} \phi$. Thus, the logic PCTL* $^{*}$ can be considered as a sublogic of POCTL*.

\subsection{The Sublogics}

An LTL formula together with a bound (QLS formula) can be interpreted over probabilistic models [2]. Recall that the logic PCTL* is a combination of PCTL

$\overline{3}$ This suffix $\sigma[1]$ is well-defined for we have previously assumed that the model does not contain any absorbing states. 
and QLS. In PCTL, arbitrary combinations of state formulas are possible, but the path formulas consists of only the next and until operators. The logic LTL allows arbitrary combinations of path formulas but only propositional state formulas. This section introduces the sublogics POCTL, OLTL and QOS of POCTL*. They can also be considered as extensions of the logics PCTL, LTL and QLS where the next operator is equipped with an observation (or a set of observations) constraint.

POCTL. We define the logic POCTL as a sublogic of POCTL* by imposing the restriction on POCTL $^{*}$ formulas that every next and until operator $(\mathbf{X}, \mathcal{U} \leq n)$ should be immediately enclosed in the probabilistic operator $\mathcal{P}$. The syntax of state and belief state formulas is the same as POCTL*, and the path formulas are given by:

$$
\phi:=\mathbf{X}_{\Omega} \Phi \mid \Phi \mathcal{U}^{\leq n} \Phi
$$

where $\Omega \subseteq \Theta$.

Since we have $\mathbf{X} \phi \equiv \mathbf{X}_{\Theta} \phi$, the logic PCTL is naturally a sublogic of POCTL. POCTL is a proper sublogic of POCTL*. For example, we let $a, a^{\prime} \in A P$, then the formulas $\mathcal{P}_{<p}(\mathbf{X X} a)$ and $\mathcal{P}_{<p}\left(a \mathcal{U}\left(\mathbf{X} a^{\prime}\right)\right)$ are not valid POCTL formulas, but are valid POCTL $^{*}$ formulas.

OLTL. In OLTL, we allow arbitrary combinations of path formulas, but only propositional state formulas. Formally, OLTL formulas are the path formulas defined by:

$$
\phi:=a|\neg \phi| \phi \wedge \phi\left|\mathbf{X}_{o} \phi\right| \phi \mathcal{U}^{\leq n} \phi
$$

QOS. Now we extend QLS to QOS (quantitative OLTL specification) which shall contribute to POCTL* model checking.

A QOS formula is a pair $(\phi, \unlhd p)$ where $\phi$ is an OLTL formula, $\unlhd \in\{\leq,<$ $, \geq,>\}$ and $p \in[0,1]$. Let $\mathcal{H}=(S, \mathbf{P}, L, \Theta, \mu, \alpha)$ be an HMM with $s \in S$. The semantics of the QOS formula is given by:

$$
\mathcal{H}, s \models(\phi, \unlhd p) \Longleftrightarrow \operatorname{Pr}_{s}(\phi) \unlhd p
$$

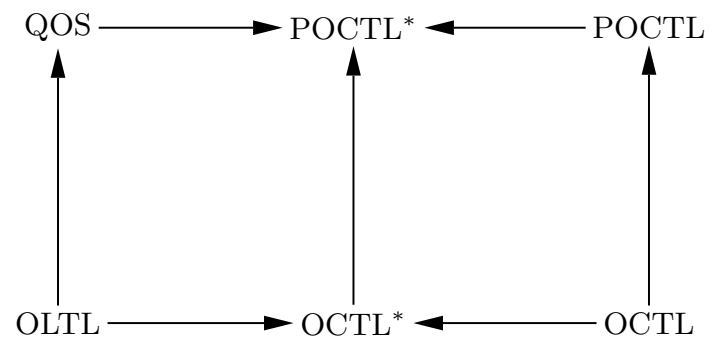

Fig. 3. Relationship of the logic POCTL* and its sublogics 
The logics OCTL* and OCTL can be defined as extensions of CTL* and $\mathrm{CTL}$, in which the next operator is equipped with an observation, and a set of observations respectively. The semantics of the sublogics are intuitively clear from the interpretation of POCTL*.

Relationship of POCTL* and Its Sublogics. Figure 3 shows an overview of the relationship of the logic POCTL* and its sublogics. There is an arrow from a $\operatorname{logic} A$ to another $\operatorname{logic} B$ if $A$ is a proper sublogic of $B$. The logics in the upper part can be considered as the probabilistic counterpart of the corresponding one in the lower part.

\subsection{Specifying Properties in POCTL*}

First, we indicate that we cannot calculate an exact probability by a POCTL* formula, however, we can specify a bound on the probability measure instead. Actually, we do not need the exact values in most cases. To illustrate the expressiveness of $\mathrm{POCTL}^{*}$, we consider following properties:

- The probability that the next observation is head and then the model goes to state fair meets the bound $<0.2$.

$$
\mathcal{P}_{<0.2}\left(\mathbf{X}_{\text {head }} \text { at }_{\text {fair }}\right)
$$

This formula can be considered as a state formula or a belief state formula. A state (belief state) satisfies this formula if the probability calculated using the measure w.r.t. the state (belief state) meets the bound $<0.2$.

- The probability is at most 0.05 , that we eventually get an observation head and then move to state fair, whereas at any moment before we are either in state $u_{1}$ or state $u_{2}$.

$$
\mathcal{P}_{\leq 0.05}\left(\left(a t_{u_{1}} \vee a t_{u_{2}}\right) \mathcal{U} \mathbf{X}_{\text {head }} a t_{\text {fair }}\right)
$$

- With probability at least 0.9 , the model generates the observation sequence $\left(o_{0}, o_{1}, \ldots, o_{n}\right)$.

$$
\mathcal{P}_{\geq 0.9}\left(\mathbf{X}_{o_{0}} \mathbf{X}_{o_{1}} \ldots \mathbf{X}_{o_{n}} t t\right)
$$

- The probability that the state sequence $\left(s_{0}, s_{1}, \ldots, s_{n}\right)$ produces the observation sequence $\left(o_{0}, o_{1}, \ldots, o_{n}\right)$ is at most 0.1 .

$$
\mathcal{P}_{\leq 0.1}\left(s_{0} \wedge \mathbf{X}_{o_{0}}\left(s_{1} \wedge \mathbf{X}_{o_{1}}\left(\ldots\left(s_{n} \wedge \mathbf{X}_{o_{n}} t t\right) \ldots\right)\right)\right)
$$

where $s$ denotes the atomic proposition that the system is now in state $s$.

\section{Model Checking}

In this section, we present model checking algorithms for the logics POCTL*, POCTL and QOS. The model checking algorithm for POCTL* follows the same 
lines as the one for PCTL* $[2,7,6]$. It will first be reduced to the QOS model checking problem. The latter can further be reduced to a probabilistic reachability analysis. To that end, we construct a Büchi automaton for a given OLTL formula. The POCTL model checking algorithm can be adapted from the one presented by Hansson \& Jonsson [11].

\subsection{POCTL* Formulas}

Let $\mathcal{H}=(S, \mathbf{P}, L, \Theta, \mu, \alpha)$ be an HMM with $s \in S$, and $\Phi$ be a POCTL* formula. The POCTL* model checking problem is to check whether $\mathcal{H}, s \models \Phi$ (or $s \models \Phi$ for short). The model checking algorithm for POCTL* is an adaption of the one presented in [2] for PCTL*.

The algorithm is based on a recursive procedure that computes the sets $\operatorname{Sat}(\Psi)$ for all state subformulas $\Psi$ of $\Phi$. The cases where $\Psi$ is an atomic proposition or a negation or a conjunction is given by: $\operatorname{Sat}(a)=\{s \in S \mid a \in L(s)\}$, $\operatorname{Sat}\left(\neg \Psi_{1}\right)=S \backslash \operatorname{Sat}\left(\Psi_{1}\right)$ and $\operatorname{Sat}\left(\Psi_{1} \wedge \Psi_{2}\right)=\operatorname{Sat}\left(\Psi_{1}\right) \cap \operatorname{Sat}\left(\Psi_{2}\right)$.

The case that $\Psi$ is the probabilistic operator $\mathcal{P}_{\unlhd p}(\phi)$ is more involved. By the semantics, it is equivalent to check whether $\operatorname{Pr}_{b_{s}}(\phi)$ meets the bound $\unlhd p$, i. e., whether $\operatorname{Pr}_{s}(\phi) \unlhd p$. Let $\Psi_{1}, \ldots, \Psi_{k}$ be the maximal state subformulas of $\phi$. The sets $\operatorname{Sat}\left(\Psi_{i}\right)$ can be calculated recursively. Then, we replace $\Psi_{1}, \ldots, \Psi_{k}$ by the new atomic propositions $n_{1}, \ldots, n_{k}$ and extend the label of state $s$ by $n_{i}$ if $n_{i} \in \operatorname{Sat}\left(\Psi_{i}\right)$.

We replace the subformulas $\Psi_{1}, \ldots, \Psi_{k}$ by new atomic propositions $n_{1}, \ldots, n_{k}$. The so obtained path formula $\phi^{\prime}$ is an OLTL formula, and obviously we have $\operatorname{Pr}_{s}(\phi)=\operatorname{Pr}_{s}\left(\phi^{\prime}\right)$. Now we apply the QOS model checking algorithm to calculate $\operatorname{Pr}_{s}\left(\phi^{\prime}\right)$, which will be discussed in Section 5.3. Hence, the complexity of the POCTL* model checking algorithm is dominated by the one for QOS.

Belief State. Now, we show how to check whether a belief state $b$ satisfies a belief state formula $\epsilon$, i. e., $b \models \epsilon$. The most interesting case is $\epsilon=\mathcal{P}_{\unlhd p}(\phi)$ where $\phi$ is a POCTL* path formula. By definition,

$$
b \models \mathcal{P}_{\unlhd p}(\phi) \Longleftrightarrow p_{b}(\phi) \unlhd p \Longleftrightarrow \sum_{s \in S} b(s) \operatorname{Pr}_{s}(\phi) \unlhd p
$$

therefore, it is sufficient to calculate $\operatorname{Pr}_{s}(\phi)$ for all $s \in S$.

\subsection{POCTL Formulas}

Let $\mathcal{H}=(S, \mathbf{P}, L, \Theta, \mu, \alpha)$ with $s \in S$, and $\Phi$ be a POCTL formula. The algorithm to check whether $s \models \Phi$ can be adapted from the one presented by Hansson \& Jonsson [11]. In case $\Phi$ is of the form $a, \neg \Phi^{\prime}, \Phi_{1} \wedge \Phi_{2}, \mathcal{P}\left(\Phi_{1} \mathcal{U} \leq n \Phi_{2}\right), \mathcal{P}\left(\Phi_{1} \mathcal{U} \Phi_{2}\right)$, the set $\operatorname{Sat}(\Phi)$ can be determined using the same strategy as for PCTL. Let $p \in[0,1], \Omega \subseteq \Theta$ and $\unlhd \in\{\leq,<, \geq,>\}$. We only need to consider the case that $\phi=\mathcal{P}_{\unlhd p}\left(\mathbf{X}_{\Omega} \Phi^{\prime}\right)$. We observe that

$$
p_{s}\left(\mathbf{X}_{\Omega} \Phi^{\prime}\right)=\mu_{s}(\Omega) \cdot \sum_{s^{\prime} \in \operatorname{Sat}\left(\Phi^{\prime}\right)} \mathrm{P}\left(s, s^{\prime}\right)
$$


where $\mu_{s}(\Omega)=\sum_{o \in \Omega} \mu_{s}(o)$ and the set $\operatorname{Sat}\left(\Phi^{\prime}\right)=\left\{s \in S \mid s \models \Phi^{\prime}\right\}$ can be recursively evaluated. Thus, $s \models \mathcal{P}_{\unlhd p}\left(\mathbf{X}_{\Omega} \Phi^{\prime}\right)$ iff $p_{s}\left(\mathbf{X}_{\Omega} \Phi^{\prime}\right) \unlhd p$.

\subsection{QOS Formulas}

This section presents the model checking algorithm for QOS formulas. We introduce two methods, an automaton based approach, which is based on the algorithm introduced by Baier et al $[2,4]$, and a direct method, where we reduce the problem to a PCTL* model checking problem over a DTMC, and apply the efficient algorithm presented by Courcoubetis et al [7].

An automaton based approach. The input is $\mathcal{H}=(S, \mathbf{P}, L, \Theta, \mu, \alpha)$ with $s \in$ $S$ and a QOS formula $(\phi, \unlhd p)$ where $p \in[0,1]$. We shall check whether $\mathcal{H}, s \models$ $(\phi, \unlhd p)$. We first construct a Büchi automaton $\mathcal{A}_{\phi}$ for $\phi$. This construction is an extension of the one presented by Gerth et al. [10] (for space reason, we present it in $[24$, Appendix A]). By the result of Safra $[18,19]$, the Büchi automaton can be translated to a deterministic Rabin automaton. Let $\mathcal{R}_{\phi}=\left(\Sigma, Q, q_{\text {in }}, \delta, U\right)$ denote the Rabin automaton for $\phi$. (Note that $\Sigma=\mathcal{P}(A P) \times \Theta$.) Next, we build the product automaton $\mathcal{H} \times \mathcal{R}_{\phi}$. Finally, the problem to calculate the measure of paths in $\operatorname{Path}^{\mathcal{H}}(s)$ satisfying $\phi$ is reduced to a probabilistic reachability analysis in the product automaton. The method we shall present is an adaption of the one introduced by Bianco \& de Alfaro [4], where we follow the presentation in [2].

The product automaton $\mathcal{H} \times \mathcal{R}_{\phi}=\left(S^{\prime}, \mathbf{P}^{\prime}, L^{\prime}\right)$ is given by: $S^{\prime}=S \times Q$, $\mathbf{P}^{\prime}\left((s, q),\left(s^{\prime}, q^{\prime}\right)\right)=\mathbf{P}\left(s, s^{\prime}\right) \cdot \mu_{s^{\prime}}(o)$ if $q^{\prime} \in \delta\left(q,\left(L\left(s^{\prime}\right), o\right)\right)$ and 0 otherwise.

For $s \in S$ and $o \in \Theta$, we define $s_{R}=\left(s, \delta\left(q_{i n},(L(s), o)\right)\right)$. Let $\sigma$ denote the path $\left(s_{0}, o_{0}\right),\left(s_{1}, o_{1}\right) \ldots$ in $\mathcal{H}$. Since $R_{\phi}$ is a deterministic automaton, we define the unique induced path $\sigma_{R}\left(s_{0}, q_{0}\right),\left(s_{1}, q_{1}\right),\left(s_{2}, q_{2}\right) \ldots$ in $\mathcal{H} \times R_{\phi}$, where $q_{0}=\delta\left(q_{i n},\left(L\left(s_{0}\right), o_{0}\right)\right), q_{i+1}=\delta\left(q_{i},\left(L\left(s_{i+1}\right), o_{i+1}\right)\right)$.

Theorem 4. Let $P_{i}^{\prime}=S \times P_{i}$ and $R_{i}^{\prime}=S \times R_{i}$. We define $U^{\prime}=\cup_{1 \leq j \leq r} U_{j}^{\prime}$, where $U_{j}^{\prime}$ is the largest subset of $P_{j}^{\prime}$ such that, for all $u^{\prime} \in U_{j}^{\prime}$ : reach ${ }^{\mathcal{H} \times R_{\phi}}\left(u^{\prime}\right) \subseteq U_{j}^{\prime}$ and reach $^{\mathcal{H} \times R_{\phi}}\left(u^{\prime}\right) \cap R_{j}^{\prime} \neq \emptyset$. Then,

$$
\operatorname{Pr}_{s}^{\mathcal{H}}(\phi)=\sum_{o \in \Theta} \mu_{s}(o) \cdot \operatorname{Pr}_{s_{R}}^{\mathcal{H} \times R_{\phi}}\left(\operatorname{reach}\left(U^{\prime}\right)\right)
$$

where $s_{R}=\left(s, \delta\left(q_{i n},(L(s), o)\right)\right)$, and $\operatorname{Pr}_{s}^{\mathcal{H}}(\phi)=\operatorname{Pr}_{s}\left\{\sigma \in \operatorname{Path}^{\mathcal{H}}(s) \mid \sigma \models \phi\right\}$ and reach $\left(U^{\prime}\right)$ denote the set of path which can reach $U^{\prime}$, i. e. $\left\{\sigma^{\prime} \in \operatorname{Path}^{\mathcal{H} \times \mathcal{R}_{\phi}}\left(s_{R}\right) \mid\right.$ $\exists i$ such that $\left.\sigma^{\prime}[i] \in U^{\prime}\right\}$.

Proof. Let $\mathcal{C}\left(\left(s, o_{0}\right),\left(s_{1}, o_{1}\right), \ldots,\left(s_{n}, o_{n}\right)\right)$ be a basic cylinder set in $\mathcal{H}$ such that every path $\sigma$ in $\mathcal{C}$ satisfies $\phi$. The measure of $\mathcal{C}$ is $\mu_{s}\left(o_{0}\right) \prod_{i=1}^{n} \mathbf{P}\left(s_{i-1}, s_{i}\right) \mu_{s_{i}}\left(o_{i}\right)$. The induced unique cylinder set in $\mathcal{H} \times \mathcal{R}_{\phi}$ is $\mathcal{C}^{\prime}\left(\left(s, q_{0}\right),\left(s_{1}, q_{1}\right), \ldots,\left(s_{n}, q_{n}\right)\right)$ where $q_{0}=\delta\left(q_{i n},\left(L(s), o_{0}\right)\right)$ and $q_{i+1}=\delta\left(q_{i},\left(L\left(s_{i+1}\right), o_{i+1}\right)\right)$ for $i=1, \ldots, n$. Obviously, $\sigma_{R}$ is in $\mathcal{C}^{\prime}$. Since $\sigma$ satisfies $\phi$, the path $\pi=q_{i n}, q_{0}, \ldots, q_{n}, \ldots$ must be an accepting path. Hence, there exists an $i$ such that $\inf (\pi) \subseteq P_{i}$ and 
$\inf (\pi) \cap R_{i} \neq \emptyset$. By the definition of $U^{\prime}, \sigma_{R}$ must contain at least one state which belongs to $U^{\prime}$.

By construction of $\mathcal{H} \times \mathcal{R}_{\phi}$, the measure of $\mathcal{C}^{\prime}$ is simply $\prod_{i=1}^{n} \mathbf{P}\left(s_{i-1}, s_{i}\right) \mu_{s_{i}}\left(o_{i}\right)$. Since $\mathcal{C}$ is an arbitrary cylinder set of interest, the above result is true for all $o_{0} \in \Theta$. Let $C_{1}, C_{2}$ be two different cylinder sets in $\mathcal{H}$. Obviously, either one cylinder set includes another, or they are disjoint. Hence, summing up over all possible observations, we are done.

Complexity. In [24, Appendix A] we show that the Büchi automaton for the OLTL formula is exponential in the size of the formula. By the results of Safra $[18,19]$, the deterministic Rabin automaton for $\phi$ is double exponential in the size of the formula. So the overall complexity of the product automaton is linear in the size of the model, and double exponential in the size of the formula.

It thus remains to compute the reachability probability $\operatorname{Pr}_{s_{R}}^{\mathcal{H} \times R_{\phi}}\left(\right.$ reach $\left.\left(U^{\prime}\right)\right)$ in the product automaton. To obtain this quantity, we can apply the method presented by de Alfaro [8, page 52]. The complexity is polynomial in the size of the product automaton.

A direct approach. The main idea of this approach is to construct a DTMC from the HMM, and transform the QOL formula $\phi$ to a QLS formula. Then, the original problem can be reduced to DTMC model checking problem.

We extend the set of atomic propositions by $A P^{\prime}=A P \cup\{\Omega \mid \Omega \subseteq \Theta\}$. Given $\mathcal{H}=(S, \mathbf{P}, L, \Theta, \mu, \alpha)$ and a QOS formula $(\phi, \unlhd p)$, we define the DTMC $\mathcal{D}=\left(S^{\prime}, \mathbf{P}^{\prime}, L^{\prime}\right)$ where $S^{\prime}=S \times \Theta, \mathbf{P}^{\prime}\left((s, o),\left(s^{\prime}, o^{\prime}\right)\right)=\mathbf{P}\left(s, s^{\prime}\right) \cdot \mu_{s^{\prime}}\left(o^{\prime}\right)$ and $L^{\prime}(s, o)=L(s) \cup\{\Omega \subseteq \Theta \mid o \in \Omega\}$. Furthermore, we define a QLS formula $\left(\phi^{\prime}, \unlhd p\right)$ as follows: Let $\mathbf{X}_{\Omega} \psi$ be a subformula of $\phi$, we replace it by $\Omega \wedge \mathbf{X} \psi$, where $\Omega$ is a new atomic proposition. We proceed this process repeatedly until there is no next formula indexed with observations.

Lemma 5. $p_{s}^{\mathcal{H}}(\phi)=\sum_{o \in \Theta} \mu_{s}(o) \cdot p_{(s, o)}^{\mathcal{D}}\left(\phi^{\prime}\right)$

Proof. Similar to Lemma 4.

Complexity. The constructed DTMC can be, in the worst case, $\mathcal{O}\left(|S|^{2}|\Theta|^{2}\right)$. We need still to calculate the probability measure of $\left\{\sigma \in \operatorname{Path}^{\mathcal{D}} \mid \sigma \models \phi^{\prime}\right\}$ in the DTMC. The optimal algorithm for that is given by Courcoubetis et al [7], and the complexity is polynomial in the size of the model, and exponential in the size of the formula.

In comparison to the other method, this method is single exponential in the size of the formula, but the DTMC suffers from the size $\mathcal{O}\left(|S|^{2}|\Theta|^{2}\right)$.

\subsection{Improving the Efficiency}

In this section, we discuss some efficiency issues for some special POCTL* formulas. After that we give some further improvements. 
The Formula $s_{0} \wedge \mathbf{X}_{o_{0}}\left(s_{1} \wedge \mathbf{X}_{o_{1}}\left(\ldots\left(s_{n} \wedge \mathbf{X}_{o_{n}} t t\right) \ldots\right)\right)$. For state $s \in S$, we let $s$ denote also the atomic propositions which asserts that the model resides in state $s$. Given a basic cylinder set $\mathcal{C}\left(\left(s_{0}, o_{0}\right), \ldots,\left(s_{n}, o_{n}\right)\right)$, we define a formula $\phi=$ $s_{0} \wedge \mathbf{X}_{o_{0}}\left(s_{1} \wedge \mathbf{X}_{o_{1}}\left(\ldots\left(s_{n} \wedge \mathbf{X}_{o_{n}} t t\right) \ldots\right)\right)$ which is called the characteristic formula of this basic cylinder set. Obviously, $\{\sigma \in$ Path $\mid \sigma \models \phi\}=\mathcal{C}\left(\left(s_{0}, o_{0}\right), \ldots,\left(s_{n}, o_{n}\right)\right)$. Hence, to check whether $s \models \mathcal{P}_{\unlhd p}(\phi)$ boils down to checking whether the probability measure of the basic cylinder set, i. e., $\operatorname{Pr}_{s}(\mathcal{C})$, meets the bound $\unlhd p$.

The Formula $\mathbf{X}_{o_{0}} \mathbf{X}_{o_{1}} \ldots \mathbf{X}_{o_{n}} t$. We define a path formula $\phi=\mathbf{X}_{o_{0}} \mathbf{X}_{o_{1}} \ldots \mathbf{X}_{o_{n}} t t$ given the cylinder set $\mathcal{C}\left(o_{0}, \ldots, o_{n}\right)=\left\{\sigma \in\right.$ Path $\left.\mid \forall i \leq n . \sigma_{o}[i]=o_{i}\right\}$. Obviously, $\{\sigma \in P$ ath $\mid \sigma \models \phi\}=\mathcal{C}\left(o_{0}, \ldots, o_{n}\right)$, which implies that to check whether $\alpha \models$ $\mathcal{P}_{\unlhd p}(\phi)$ boils down to checking whether $\sum_{s \in S} \alpha(s) \operatorname{Pr}_{s}(\mathcal{C})$ meets the bound $\unlhd p$. The value $\operatorname{Pr}_{s}(\mathcal{C})$ can be calculated using Forward-Backward method presented in [17], with complexity $\mathcal{O}\left(|S|^{2} n\right)$.

Building the Automaton by Need. The set of states of the product automaton contains all pairs $(s, q) \in S \times Q$. In case $\Phi$ is a simple probabilistic operator, i. e., $\mathcal{P}_{\triangleleft p}(\phi)$ where there is no probabilistic operator in $\phi$, we only need the states of the product automaton which are reachable from initial states $s_{R}$. So in this case we can construct the states of the product automaton as needed.

Reducing to POCTL Model Checking. Since the POCTL model checking algorithm is more efficient, we can use it to deal with QOS formulas of the form $(\phi \mathcal{U} \psi, \unlhd p)($ or $(\phi \mathcal{U} \leq n \psi, \unlhd p))$ where $\phi$ and $\psi$ are POCTL $^{*}$ path formulas which can be verified recursively.

\section{Conclusion and Future Work}

\subsection{Conclusion}

In this paper, we have defined probability spaces (w.r.t. state and belief state) for a given HMM. We have presented the temporal logic POCTL* with which we can specify state-based, path-based and belief state-based properties over HMMs. With POCTL* one can specify properties not only over the underlying DTMC, but also over the set of processes producing observations. Finally, we have focused on the POCTL* model checking algorithm. The most interesting case is to deal with the probabilistic operator, and we have shown that this can be reduced to QOS model checking. Then, the QOS model checking problem is reduced to a probabilistic reachability analysis in the product automaton of the HMM and a deterministic Rabin automaton. The complexity of our model checking algorithm is polynomial in the size of the model and exponential in the length of the formula.

\subsection{Future Work}

In this section, we consider some interesting directions for future work. 
$H M D P$. We plan to extend an HMM to a Hidden Markov decision process (HMDP) [4,8] where probabilistic and nondeterministic choices coexist. In an HMM, a successor of a state $s$ is selected probabilistically according to the transition matrix. On the contrary, in an HMDP, for a state $s$, one first selects a probabilistic distribution over actions nondeterministically. Then, a successor can be chosen probabilistically according to the selected distribution over actions.

The nondeterminism is resolved by schedulers [3] (called strategy in [4,8], adversary in [2]). A scheduler $\eta$ assigns a distribution over actions to a finite sequence of states (history). Given a scheduler $\eta$, one can select a successor of a state probabilistically, as in an HMM. Moreover, we can get a probability measure [4] $\operatorname{Pr}_{s}^{\eta}$ w.r.t. the scheduler $\eta$ and a state $s$. Thus, the logic POCTL* can be extended to interpret properties over HMDPs in the following way:

$$
s \models \mathcal{P}_{\unlhd p}(\phi) \quad \text { iff } \quad \forall \eta \cdot \operatorname{Pr}_{s}^{\eta}\left\{\sigma \in P a t h^{\eta} \mid \sigma \models \phi\right\} \unlhd p
$$

Since a belief state is a distribution over states, we can extend the probability measure w.r.t. $s$ and $\eta$ to the one w.r.t. a belief state and $\eta$. The semantics that a belief state satisfies a belief state formula can also be defined in a similar way. The model checking algorithm can be adapted from the one presented by de Alfaro for PCTL* formulas over MDPs.

HMDP with Fairness. Baier [2] extended the logic PCTL* to interpret properties over concurrent probabilistic systems (similar to MDPs) with fairness assumptions. She also presented a PCTL* model checking algorithm over concurrent probabilistic systems with fairness assumptions which is adapted from the one by de Alfaro. It could be extended to a POCTL* model checking algorithm over HMDPs with fairness assumptions.

Acknowledgements. The authors are grateful to Christel Baier (University of Bonn) and Frits Vaandrager (Radboud University Nijmegen) for helpful comments at an early state of the work presented in this paper.

\section{References}

1. Suzana Andova, Holger Hermanns, and Joost-Pieter Katoen. Discrete-time rewards model-checked. In FORMATS, LNCS 2791:88-104. Springer, 2003.

2. C. Baier. On Algorithmic Verification Methods for Probabilistic Systems, 1998. Habilitations- schrift zur Erlangung der venia legendi der Fakultät für Mathematik and Informatik, Universität Mannheim.

3. C. Baier, B.R. Haverkort, H. Hermanns, and J.-P. Katoen. Efficient computation of time-bounded reachability probabilities in uniformized continuous-time Markov decision processes. In TACAS, LNCS 2988:61-76. Springer, 2004.

4. A. Bianco and L. de Alfaro. Model Checking of Probabilistic and Nondeterministic Systems. In FSTTCS, LNCS 1026:499-513. Springer, 1995.

5. E. Birney. Hidden Markov models in biological sequence analysis. IBM Journal of Research and Development, 45(3):449-454, 2001. 
6. C. Courcoubetis and M. Yannakakis. Verifying Temporal Properties of FiniteState Probabilistic Programs. In FOCS:338-345. IEEE Computer Society Press, October 1988.

7. C. Courcoubetis and M. Yannakakis. The Complexity of Probabilistic Verification. Journal of the ACM, 42(4):857-907, 1995.

8. L. de Alfaro. Formal Verification of Probabilistic Systems. PhD thesis, Stanford University, 1997. Technical report STAN-CS-TR-98-1601.

9. J.-M. François and G. Leduc. Mobility prediction's influence on QoS in wireless networks: A study on a call admission algorithm. In 3rd International Symposium on Modeling and Optimization in Mobile, Ad-Hoc and Wireless Networks, pages 238-247. IEEE Computer Society, 2005.

10. R. Gerth, D. Peled, M.Y. Vardi, and P. Wolper. Simple On-the-fly Automatic Verification of Linear Temporal Logic. In PSTV 38:3-18. Chapman \& Hall, 1995.

11. H. Hansson and B. Jonsson. A Logic for Reasoning about Time and Reliability. Formal Aspects of Computing, 6(5):512-535, 1994.

12. M. Hauskrecht. Value-Function Approximations for Partially Observable Markov Decision Processes. Journal of Artificial Intelligence Research, 13:33-94, 2000.

13. D. Jurafsky and J.H. Martin. Speech and Language Processing: An Introduction to Natural Language Processing, Computational Linguistics, and Speech Recognition. Prentice Hall, 2000.

14. R. D. Nicola and F. W. Vaandrager. Action versus state based logics for transition systems. In Semantics of Systems of Concurrent Processes, LNCS 469:407-419. Springer, 1990.

15. P.A. Pevzner. Computational Molecular Biology: An Algorithmic Approach. The MIT Press, 2000.

16. P. Poupart. Approximate Value-Directed Belief State Monitoring for Partially Observable Markov Decision Processes. Master's thesis, University of British Columbia, November 2000.

17. L.R. Rabiner. A Tutorial on Hidden Markov Models and Selected Applications in Speech Recognition. Proceedings of the IEEE, 77(2):257-286, February 1989.

18. S. Safra. On the complexity of $\omega$-automata. In FOCS, pages 319-327, 1988.

19. S. Safra. Exponential determinization for $\omega$-automata with strong-fairness acceptance condition. In STOC, pages 275-282, 1992.

20. K. Salamatian and S. Vaton. Hidden markov modeling for network communication channels. In SIGMETRICS, pages 92-101. ACM Press, 2001.

21. M. Y. Vardi and P. Wolper. An Automata-Theoretic Approach to Automatic Program Verification. In $L I C S$, pages 332-345. IEEE Computer Society Press, June 1986.

22. J. A. Vlontzos and S. Y. Kung. Hidden Markov models for character recognition. IEEE Transactions on Image Processing, 1:539-543, October 1992.

23. P. Wolper, M. Y. Vardi, and A. P. Sistla. Reasoning about Infinite Computation Paths. In FOCS '83, pages 185-194. IEEE Computer Society Press, 1982.

24. L. Zhang, H. Hermanns, and D. N. Jansen. Logic and Model Checking for Hidden Markov Chais. AVACS Technical Report No. 6, SFB/TR 14 AVACS, May 2005. ISSN: 1860-9821, http://www.avacs.org. 